Document downloaded from:

http://hdl.handle.net/10251/45036

This paper must be cited as:

Sendra Compte, S.; Lamparero Arroyo, JV.; Lloret, J.; Ardid Ramírez, M. (2012). Study of the Optimum Frequency at 2.4GHz ISM Band for Underwater Wireless Ad Hoc Communications. En Lecture Notes in Computer Science. Springer Verlag (Germany). 260273. doi:10.1007/978-3-642-31638-8_20.

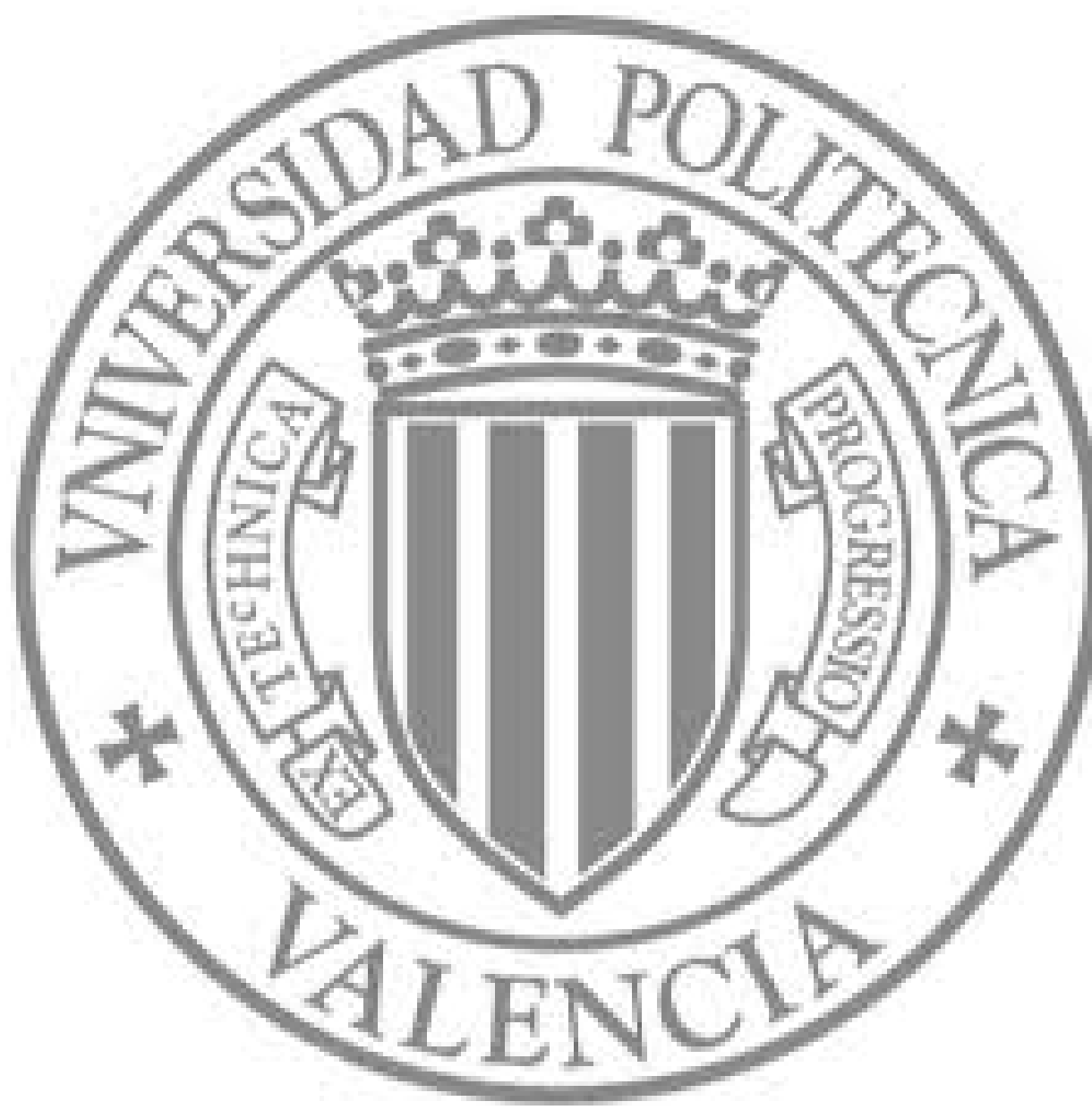

The final publication is available at

http://dx.doi.org/10.1007/978-3-642-31638-8_20

Copyright Springer Verlag (Germany) 


\title{
Study of the Optimum Frequency at 2.4GHz ISM Band for Underwater Wireless Ad Hoc Communications
}

\author{
Sandra Sendra, Jose V. Lamparero, Jaime Lloret and Miguel Ardid \\ Instituto de Investigación para la Gestión Integrada de zonas Costeras \\ Universidad Politécnica de Valencia \\ 46730 Grao de Gandia (Valencia), Spain \\ sansenco@posgrado.upv.es, josevi@lamparero.es, jlloret@dcom.upv.es, mardid@fis.upv.es
}

\begin{abstract}
Underwater communications at low frequencies are characterized by the low data rate. But in some cases wireless sensors must be placed quite close to each other and need high data rates in order to accurately sense an ecosystem that could be contaminated by invasive plants or hazardous waste. Most researchers focus their efforts on increasing the data transfer rates for low frequencies, but, due to the wave features, this is very complicated. For this reason, we propose the use of high frequency band communications for these special cases. In this paper we measure the optimum working frequency for an underwater communication in the $2.4 \mathrm{GHz}$ range. We measure the number of lost packets and the average round trip time value for a point-to-point link for different distances. These measures will be performed by varying the data rate, the type of modulation and the working frequency. We will show that we are able to transmit higher data transfer rates, by using higher frequencies, than the using acoustic waves.
\end{abstract}

Keywords: Underwater Wireless Ad Hoc Communications; 2.4 GHz; UWSN.

\section{Introduction}

Research related to underwater communications and ad-hoc networks are growing rapidly. One of the main research lines that are being studied, in ad-hoc networks, is the increase of the network lifetime $[1,2]$. When we try to implement ad hoc underwater network, we encounter other problems, such as, the low performance of underwater communication systems.

Communication systems based on optical waves and acoustic techniques are being used in wireless communication deployments for underwater environments. But both transmission systems have advantages and disadvantages [3]. On one hand, the systems that are able to reach very high propagation speed are those based on optical communication. However, due to the suspended particles and the turbidity of the water, this system presents a strong backscattering, so it is not good option for long distances. On the other hand, systems based on acoustic waves are not so sensible to 
suspended particles and turbidity of water. Low frequencies are used in these kinds of systems, so there are problems with latency. Moreover, there is a low data rate.

Electromagnetic (EM) waves, in the RF range, can also be used for underwater wireless communication systems as a good option. These waves are less sensitive to reflection and refraction effects in shallow water, than acoustic waves. Moreover, suspended particles have very little impact on them. The speed of EM waves in the water is $2,25 \times 10^{8} \mathrm{~m} / \mathrm{s}$, meanwhile the speed of acoustic waves is around $1500 \mathrm{~m} / \mathrm{s}$. This parameter depends mainly on 4 environmental factors, which are: permeability $(\mu)$, permittivity $(\varepsilon)$, conductivity $(\sigma)$ and volume charge density $\rho$ [4]. But there are some effects that can change the water nature. The wave propagation speed and absorption coefficient vary as a function of the presence of dissolved salts in water, which changes the electrical conductivity value associated to the medium. The conductivity is directly related to the working frequency. Conductivity presents different values for each case. Seawater has a conductivity average value around 4 $\mathrm{S} / \mathrm{m}$ (this value changes depending on the tested sea), but in fresh water the typical value is $0.01 \mathrm{~S} / \mathrm{m}$ (400 times less) and drinking water presents a conductivity around $0.005-0.05 \mathrm{~S} / \mathrm{m}$. References [5] and [6] show a relationship model that relates the changes of the frequency with the temperature, the salinity, and the permittivity of the seawater. Thus, the main problem of underwater communication with EM waves is the high attenuation, due to the conductivity of the water, and its increase when the frequency of EM waves increases. For this reason, the higher frequencies always register higher attenuation losses. Considering all these factors, we performed a practical study in underwater environments. We tested the behavior of EM signals in this medium. In order to perform it we used devices compatible with IEEE 802.11 standard [7].

This paper addresses the tests performed at different frequencies and modulations in order to check various parameters such as minimum depth, distance between devices and signal transmission characteristics. Tests have been performed in the first seven frequencies (specified in the IEEE 802.11 standard), that correspond with the frequency range from $2.412 \mathrm{GHz}$ to $2.442 \mathrm{GHz}$. We performed and ad hoc communication between two devices, a Personal Computer (PC) and an access point (AP), in order to monitor the activity of the underwater point-to-point link. We have used the echo request and echo reply packets in order to perform our tests. The high attenuation given at these frequencies leaded us to think that underwater communications at $2.4 \mathrm{GHz}$ band is unhelpful and impractical, but as we shall see at the end of this paper, there are many applications where the use of this technology will bring many benefits.

The rest of the paper is structured as follows. We finish the first section, showing some previous work. Section 2 overviews some aspects about the used modulations and data rate of IEEE $802.11 \mathrm{~b} / \mathrm{g}$. Section 3 describes the scenario, hardware and software used in order to take the right measurements. The performance results are presented in Section 4. Finally, Section 5 shows the conclusion and future work. 


\subsection{Related Work}

The most widely used waves in underwater communications are the acoustic waves. There is a huge variety of articles, which describe and propose underwater communications systems. However it is not so common the description of underwater communications systems using acoustic waves.

In [8], Chaitanya et al. show an example of path loss analysis given by the reflection and refractions. Moreover, we can see the effects of depth and temperature in this type of waves [9]. For systems based on optical communications, we can also find a great variety of studies about their propagation and losses [10].

As far as we know, due to the limited use of EM in underwater environments, there is very few literature published about them. We can find some generic papers, where the authors show the mathematical formulation that should be taken into account when working with EM waves [11]. One of them is the paper authored by Jiang et al. in [12]. They conducted a study of the EM wave's propagation in fresh water for frequencies between $23 \mathrm{kHz}$ and $1 \mathrm{GHz}$. They also analyzed other parameters that are related to the waves transmission speed.

In previous experiments [13], the authors of this paper performed a study on RF communication in the $2.4 \mathrm{GHz}$ ISM frequency band. We demonstrated that it is a feasible option to use the EM waves in order to establish an underwater wireless link and to transmit high data bit rates between two devices. We performed several tests for 1, 2, 5.5 and $11 \mathrm{Mbps}$ at different frequencies.

Except the last paper presented in this section, which is our paper, we have not found any other paper in the related literature showing the performance of underwater communication tests at $2.4 \mathrm{GHz}$.

\section{Modulations and Data Rate Overview}

This section shows the parameters taken into account in our measurements: the modulation type and the data rates. We have analyzed another technology that use the same frequency as IEEE 802.11 [7]. It is the IEEE 802.15.4 standard [14]. Moreover, because our tests were performed using commercial devices, operating under the IEEE $802.11 \mathrm{~b} / \mathrm{g}$, we also discuss the standard and identify each type of modulation with the data rates specified in the standard.

IEEE 802.11 standard defines the value of maximum data transfer rates depending on the used modulation. Each variant can be chosen depending on the system where it is going to be applied. In our experiments, we used the Phase-Shift Keying (PSK) [15] and the Complementary Code Keying (CCK) [16] modulations. CCK and PKS modulations operate at a theoretical data rates up to $11 \mathrm{Mbps}$ in the range of 2.400 $\mathrm{GHz}$ to $2.4835 \mathrm{GHz}$. BPSK and QPSK modulations are optimal from the error protection point of view. BPSK is used for low-cost transmitters that do not require high speeds. CCK modulation allows encoding multiple bits of data directly on a single chip with eight 64-bit sequences. Therefore, CCK method can achieve a maximum speed of $5.5 \mathrm{Mbps}$ by encoding 4 bits at a time or up to $11 \mathrm{Mbps}$ by encoding 8 bits of data. 
Fig. 1 shows a comparison of the maximum data transfer rates of both wireless technologies. It shows that devices that use IEEE 802.15.4 standard have much lower data transfer rates than IEEE 802.11 standard.

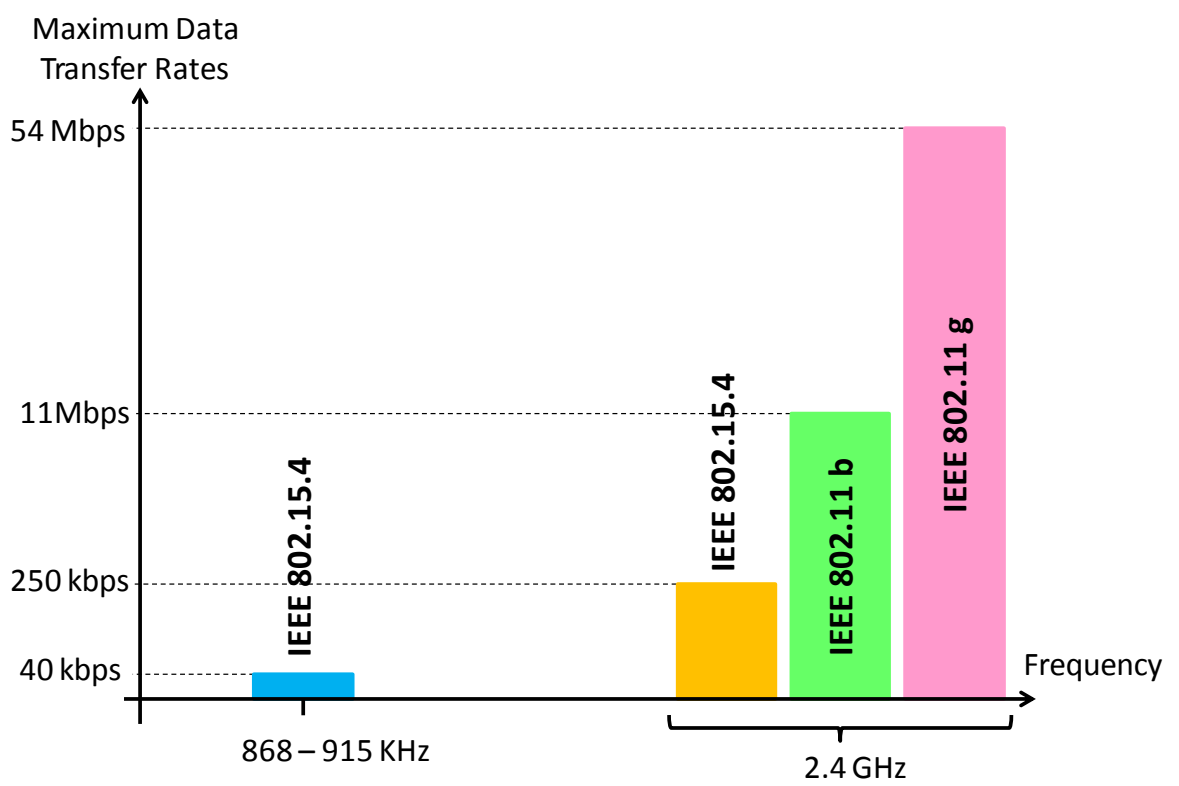

Fig. 1. Data transfer rates comparison of some wireless technologies.

Although IEEE 802.15.4 has lower power consumption than the other technology, as we can see in figure 2, it also presents low data transfer rates. Our application needs data transfer rates higher than the ones offered by IEEE 802.14.5. For this reason, we have decided to sacrifice a little the power consumption in favor of enhancing the system data rates.

Table 1 identifies the used modulations and the maximum data rates for IEEE $802.11 \mathrm{~b} / \mathrm{g}$ variants. In order to take measurements, first we determined the distance between devices were the number of delivered packets without errors is higher than $50 \%$ at least. We observed at $15.5 \mathrm{~cm}$ that the percentage of packets delivered successfully is quite high, while for $16 \mathrm{~cm}$, these values begin to decrease. Then we measure the number of lost packets and the round trip time (RTT) value for each type of modulation and transfer rate for each frequency. We will also do the test for $17 \mathrm{~cm}$, where these values are very low, as we saw in [13]. With these measures, we aim to see if varying the frequency and modulation scheme, we obtain better results. In order to determine which modulation and transmission schemes are good to be added in our tests, we performed some preliminary tests. We found that the OFDM transmission scheme presented worse behavior than the other three modulations (BPSK, QPSK and CCK). That is why we did not include it in our test. Table 1 shows data transfer rates for BPSK, QPSK and CCK. 
Table 1. Modulations and data rates used in IEEE $802.11 \mathrm{~b} / \mathrm{g}$.

\begin{tabular}{lcccc}
\hline Modulation & BPSK & QPSK & CCK & CCK \\
\hline Data rates & $1 \mathrm{Mbps}$ & $2 \mathrm{Mbps}$ & $5.5 \mathrm{Mbps}$ & $11 \mathrm{Mbps}$ \\
\hline
\end{tabular}

\section{Scenario, Hardware and Measurements strategies}

This section describes the scenario where measures have been taken and the hardware and software used for our tests. It also explains the preliminary performed tests.

\subsection{Place to take measurements}

We have placed the system in a swimming pool which has $32 \mathrm{~m}^{2}$ surface (it as 8 meters length and 4 meters wide). It has a depth between $1.5 \mathrm{~m}$ and $1.80 \mathrm{~m}$ (depending on the side) and the brick walls are covered with small mosaic tiles. Because the swimming pool dimensions are much greater than the distance which the devices are located, we will avoid any reflection and refraction on the walls, ground and surface water (due to the change of medium). The measurements were taken in fresh water with a temperature of $26{ }^{\circ} \mathrm{C}$. The $\mathrm{pH}$ value was 7.2 and the amount of chlorine and bromine dissolved in the water was $0.3 \mathrm{mg} / \mathrm{l}$.

Fig. 2 shows the sketch of the swimming pool used to perform our measurements.

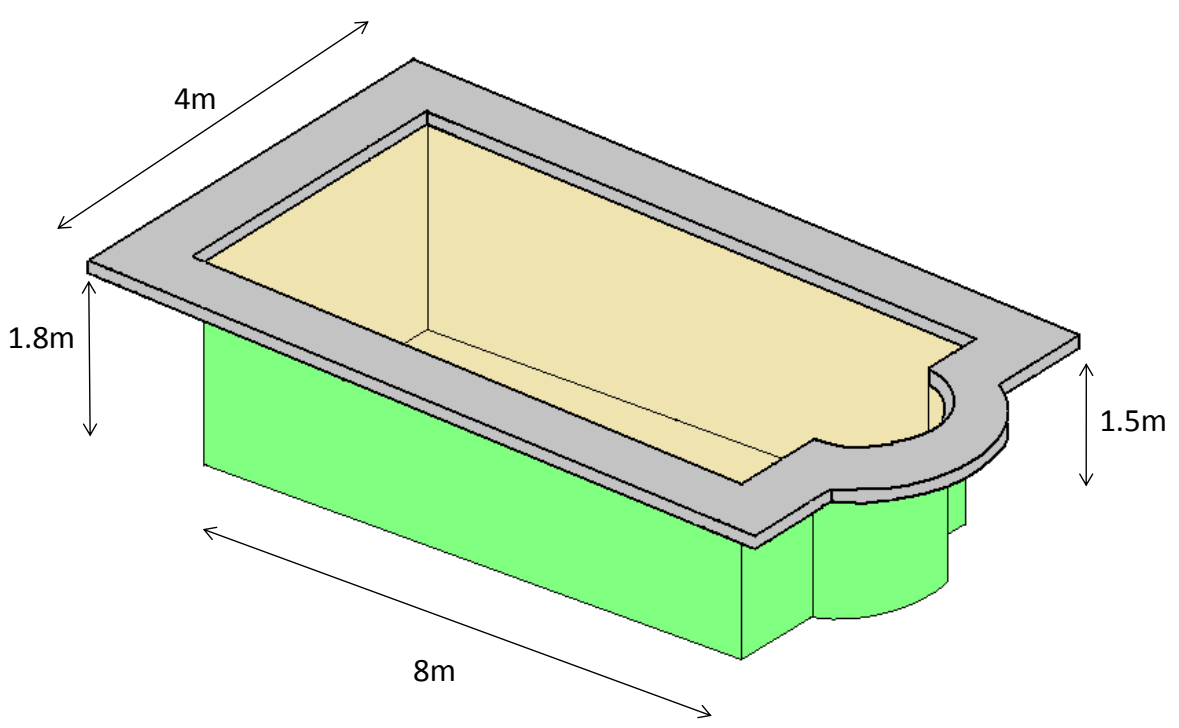

Fig. 2. Swimming pool where measures have been taken. 


\subsection{Elements used in the tests}

In order to perform the tests we used a wireless AP Dlink DWL-2100AP. This AP can work under IEEE $802.1 \mathrm{~b} / \mathrm{g}$. It can be configured to work as a wireless AP, as a bridge for a point to point connection with another wireless bridge, as a bridge for a point-multipoint connection with another bridge or as a wireless client. Its output power is around the $16 \mathrm{dBm}$.

The AP uses a vertical monopole antenna with $2 \mathrm{dBi}$ of gain. It is an antenna consisting of a single radiating arm straight vertically. This antenna has to be completed by a ground plane to operate properly. This ground plane can be natural (a water surface to facilitate electron conduction) or artificial (a number of drivers who are joined at the base of the monopole).

We also used a laptop (located outside the water) as a second device to monitor the wireless network from outside the water. In order to connect the antennae which are placed inside the water, to the devices which are outside, we used 2 pigtails of 3 meters.

Fig. 3 shows the topology of the test bench. It shows the AP, the computer and the two antennas inside the water.

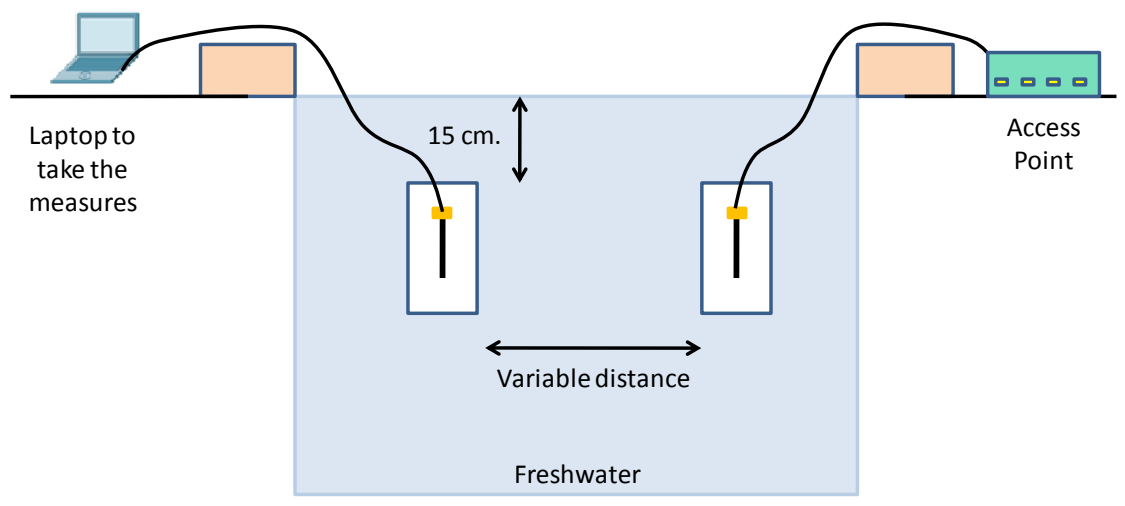

Fig. 3. Measurement setup

In order to take the measurements, we used the same method used to check the status of a network connection. Concretely, we used some common commands in the command-line shell interface. It let us sent a continuous packet flow, using the echo request and echo reply packets. Then, we collected and analyzed the obtained results. From these data, it is easy to extract the system performance in terms of communication distance, data transfer rate, average RTT and \% of lost packets for each frequency. It is only needed a simple data processing.

\subsection{Measurement strategies and scenario preparation}

First of all, it is important to ensure that the measurements taken are valid and the signal did not spread out of the water. Then, the first step was to determine the 
minimum depth where the antennae should be placed. We introduce the AP antenna in the water and we established an ad hoc wireless connection between the PC and the AP. When the laptop placed outside did not get any signal from antenna AP that was introduced in the water, we had obtained the minimum depth. We lost the signal from the AP when it is at $15 \mathrm{~cm}$. deep. With this simple test, we were ensuring that the only signal received by the laptop is provided by the antenna placed inside the water.

In the second test, we check the effect of different power emissions. We tested different values of power, between $100 \mathrm{~mW}$ and $800 \mathrm{~mW}$. Interestingly and contrary to what happens when we work with these devices in the air, increasing the transmission power, the maximum distance between devices, does not increase. In addition, we observed that the transmission behavior worsens. We therefore decided to work at $100 \mathrm{~mW}$.

In the third preliminary test, we checked if the antenna emits when it is in contact directly with water. In this case, the antennas were sealed and plunged into the water. We observed that the antennas had to be very close to each other (almost touching). Therefore, we decide to put them in a watertight container, so the antenna could start emitting into the air and then the signal propagates through the water. We also tested the effect of container size of the antenna. By different studies on wireless signal propagation and path loss [17], we know that the greatest signal strength is found just one meter from the sending device. From this point, signal starts to decrease. We wanted to see if this is also repeated in the water. To do this, we take a container with a length of $1.5 \mathrm{~m}$ and the antenna is situated inside, so that they had a 1 meter on the one side and $0.5 \mathrm{~m}$ on the other side. Both antennas were submerged in the water and we checked the maximum distance between the two antennas, without reducing the network performance. Several container sizes were checked and we saw that the performance does not improve, when distance between antennas increases. Finally, we used small containers of $5 \mathrm{~cm}$ in diameter and the distance is the same. Therefore, we conducted tests with small containers.

Several tests were conducted in the frequency range between $2.412 \mathrm{GHz}$ and 2.472 GHz. These values correspond to the spectrum used by devices that work under the IEEE $802.11 \mathrm{~b} / \mathrm{g}$. These tests allow us to characterize the behavior of an underwater communication, based on EM waves, which will allow high transfer data rates.

\section{Performance Results}

This section shows the obtained results. We have tested several frequencies specified in the IEEE 802.11 standard. These frequencies are $2.412 \mathrm{GHz}, 2.417 \mathrm{GHz}, 2.422$ $\mathrm{GHz}, 2.427 \mathrm{GHz}, 2.432 \mathrm{GHz}, 2.437 \mathrm{GHz}$ and $2.442 \mathrm{GHz}$. For higher frequencies the value of lost packets is around $90-100 \%$, which is a very bad value for a communications system.

We analyzed the variation of the RTT between both devices, depending on the distance between the antennas. We also measured the amount of lost packets and the communication behavior, depending on the type of modulation. Each test was 3 minutes long. We distinguish two types of packets: packets successfully received and packets which were not received or were received wrong. For the second type of 
packets, we assigned the value of 3,000 ms. In this way, we denoted that no echo will be received for that cases. We know this due to the wave propagation speed through water and the distance between both antennas. We measured the behavior of the BPSK, QPSK and CCK modulations with data transfer rates up to $1 \mathrm{Mbps}, 2 \mathrm{Mbps}$, 5.5 Mbps and 11 Mbps.

\subsection{Measures for $1 \mathrm{Mbps}$}

Fig. 4 shows the percentage of lost packets for a data transmission rate of $1 \mathrm{Mbps}$ using BPSK modulation. The frequencies that recorded the highest lost packets values were $2.427 \mathrm{GHz}, 2.437 \mathrm{GHz}$ and $2.442 \mathrm{GHz}$, for a distance of $16 \mathrm{~cm}$, while the highest losses for a distance of $17 \mathrm{~cm}$ are registered at $2.417 \mathrm{GHz}, 2.437 \mathrm{GHz}$ and $2.442 \mathrm{GHz}$.

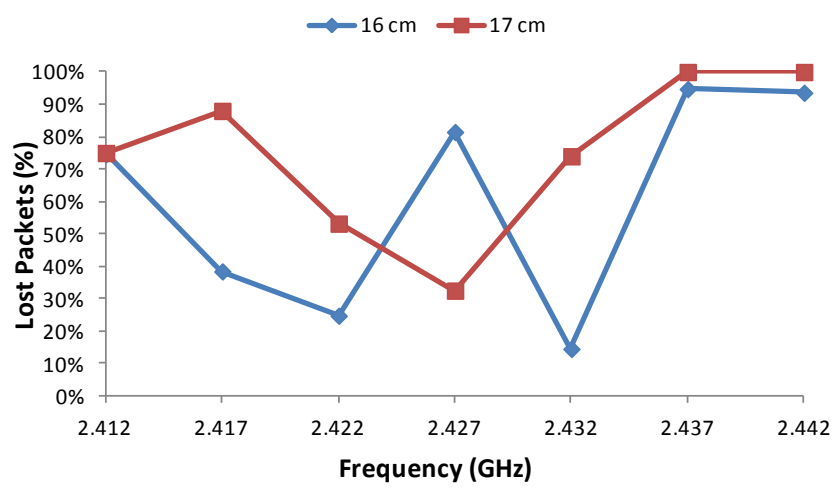

Fig. 4. Lost packets for 1 Mbps data rate.

Fig. 5 shows the average RTT values in milliseconds for 1 Mbps data transfer rates, when BPSK modulation is used. The average RTT for both distances is relatively small (around $20 \mathrm{~ms}$ ). In $2.437 \mathrm{GHz}$ the RTT value for $16 \mathrm{~cm}$ increases up to $500 \mathrm{~ms}$, while for $17 \mathrm{~cm}$ there are not packets registered and the RTT obtained is $3,000 \mathrm{~ms}$.

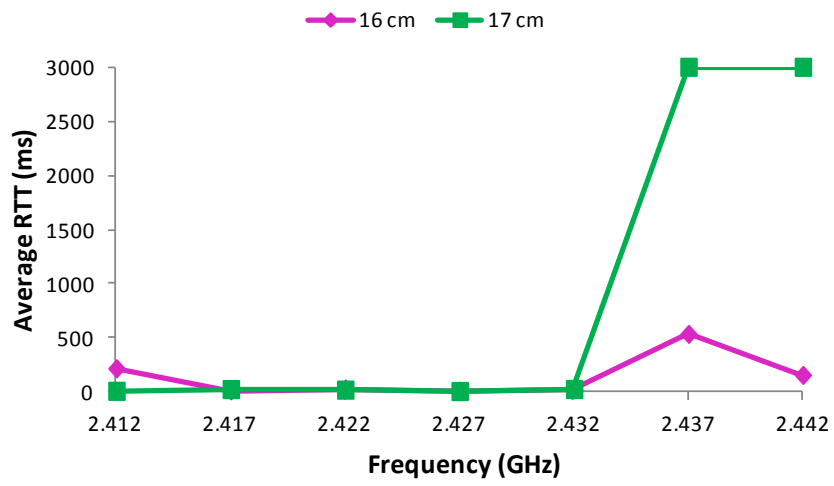

Fig. 5. Average RTT for 1 Mbps data rate. 


\subsection{Measures for 2 Mbps}

Fig.6 shows the percentage of lost packets for $2 \mathrm{Mbps}$ data transfer rates, when QPSK modulation is used. In this case, the frequencies with the lowest lost packets percentage are $2.412 \mathrm{GHz}, 2.427 \mathrm{GHz}$ and $2.437 \mathrm{GHz}$, for a distance of $16 \mathrm{~cm}$, while for a distance of $17 \mathrm{~cm}$ the lowest losses are given at $2.422 \mathrm{GHz}$.

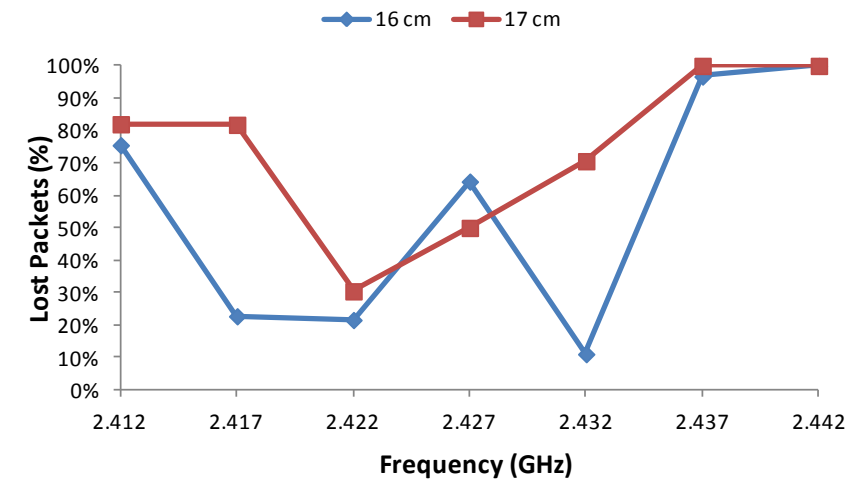

Fig. 6. Lost packets for $2 \mathrm{Mbps}$ data rate.

Fig. 7 shows the average RTT, in milliseconds, for 2 Mbps data transfer rates when QPSK modulation is used. The average RTT values for both distances are kept below $500 \mathrm{~ms}$ for a frequency of $2.432 \mathrm{GHz}$. For a distance of $16 \mathrm{~cm}$, the average RTT value at $2.437 \mathrm{GHz}$ is around $900 \mathrm{~ms}$ and, finally, this value increases up to 3,000 ms, at the same frequency, for a distance of $17 \mathrm{~cm}$.

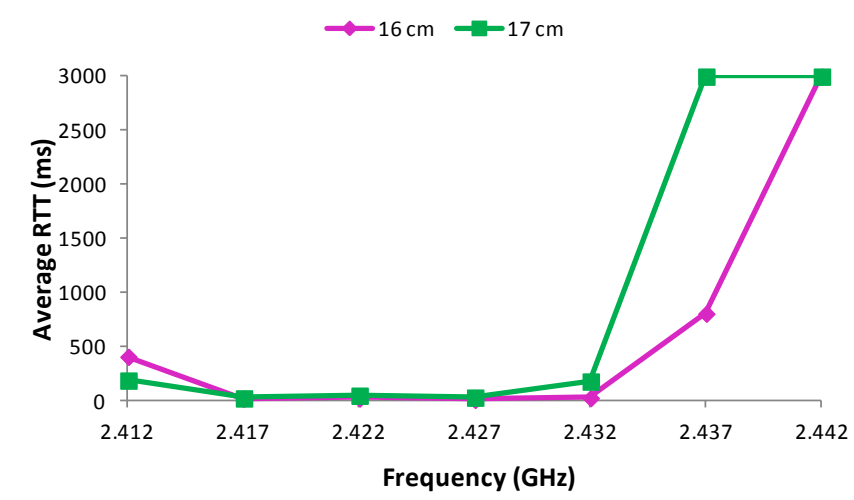

Fig. 7. Average RTT for 2 Mbps data rate.

\subsection{Measures for 5.5 Mbps}

Fig. 8 shows the percentage of lost packets for 5.5 Mbps data transfer rates when CCK modulation is used. We can see that the lost packets percentage has worsened almost threefold at $2.412 \mathrm{GHz}$ and $2.417 \mathrm{GHz}$ for both distances. In addition, for 16 
$\mathrm{cm}$, only $2.412 \mathrm{GHz}$ and $2.417 \mathrm{GHz}$ frequencies had losses below $50 \%$, meanwhile, for $17 \mathrm{~cm}$, the frequency that registers the lowest lost packets percentage is 2.427 GHz.

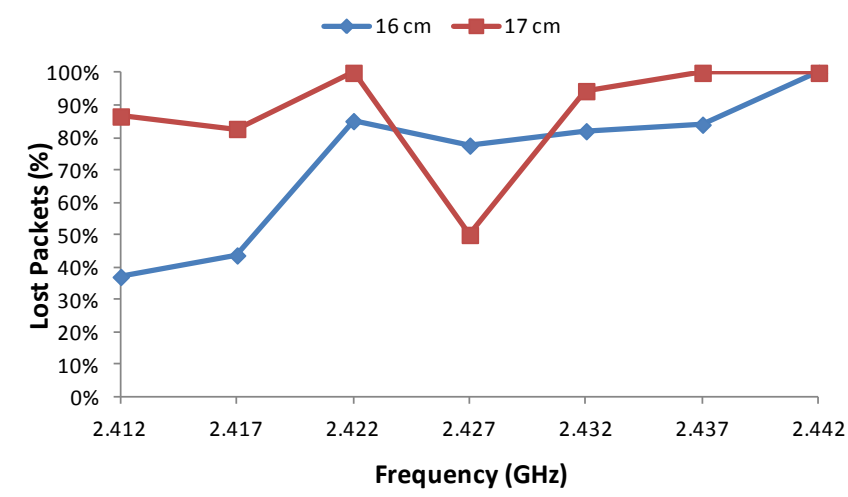

Fig. 8. Lost packets for 5.5 Mbps data rate.

Fig. 9 shows the average RTT, in milliseconds, for 5.5 Mbps data transfer rate, using CCK modulation. In this case, the RTT values for both distances are less than $500 \mathrm{~ms}$ from $2.412 \mathrm{GHz}$ to $2.432 \mathrm{GHz}$, while at $2.437 \mathrm{GHz}$ the RTT value increases above to $2,000 \mathrm{~ms}$ in both cases.

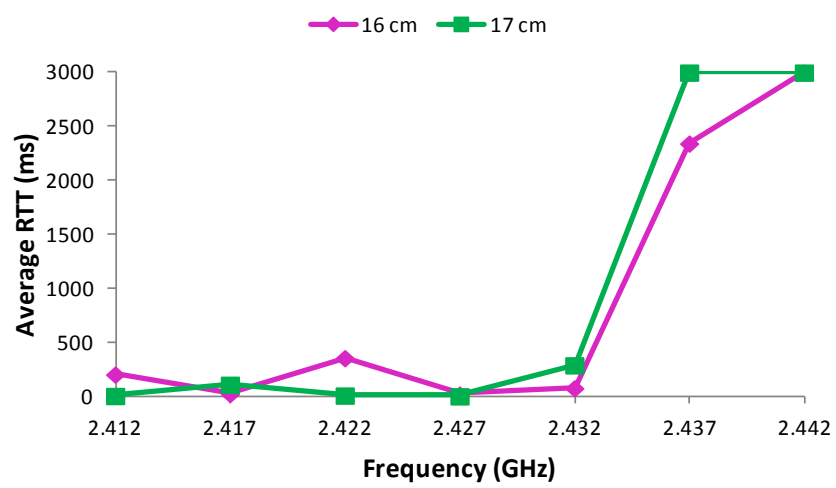

Fig. 9. Average RTT for5,5 Mbps data rate.

\subsection{Measures for 11 Mbps}

Fig. 10 shows the percentage of lost packets for $11 \mathrm{Mbps}$ data rate, when CCK modulation is used. We see that the percentage of lost packets for $16 \mathrm{~cm}$ increase almost linearly with the frequency. Just the amount of lost packets for $2.412 \mathrm{GHz}$ and $2.417 \mathrm{GHz}$, are below $70 \%$. Analyzing the behavior of all frequencies for $17 \mathrm{~cm}$, the system presents lost packets values above $70 \%$. 


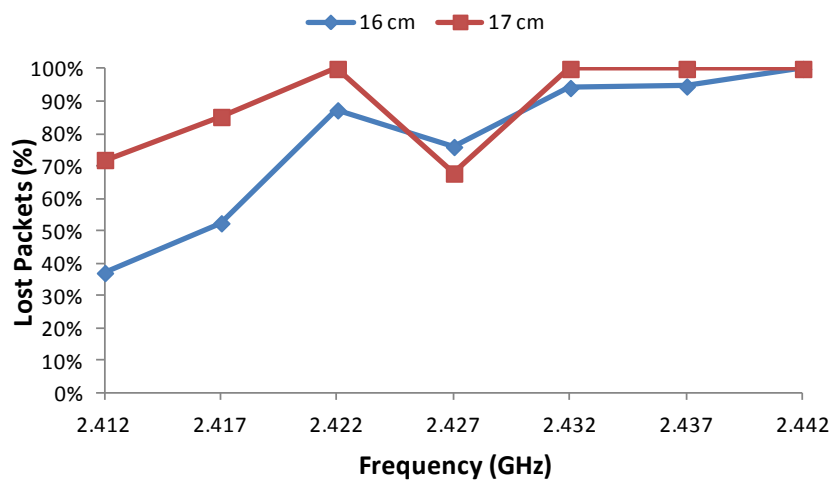

Fig. 10. Lost packets for $11 \mathrm{Mbps}$ data rate.

Fig. 11 shows the average RTT in milliseconds for $11 \mathrm{Mbps}$ data transfer rates, when CCK modulation is used. The average RTT values obtained for $16 \mathrm{~cm}$ remain around 400-600 ms at frequencies below $2.437 \mathrm{GHz}$, while in $2.442 \mathrm{GHz}$ we did not receive any packet. In $17 \mathrm{~cm}$, the obtained average RTT values are very low for 2.412 $\mathrm{GHz}, 2.417 \mathrm{GHz}$ and $2.427 \mathrm{GHz}$, but it reached 3,000 ms. for all other frequencies.

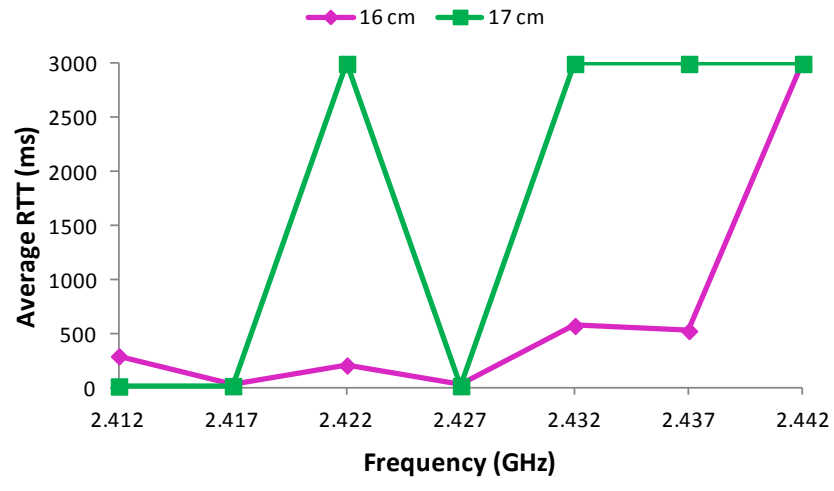

Fig. 11. Average RTT for $11 \mathrm{Mbps}$ data rate.

\subsection{Summary of results}

After having produced and presented all the results of our measurements, we summarize in this section the values obtained.

Table 2 shows a summary for a distance of $16 \mathrm{~cm}$, with the best results of each case of the measurements previously shown. It specifies the frequencies that showed the lowest lost packets values and the average RTT values in milliseconds. Table 3 shows a summary of the same values, for a distance of $17 \mathrm{~cm}$. 
Table 2. Summary of results for $16 \mathrm{~cm}$.

\begin{tabular}{llll}
\hline Modulation & Best frequencies $(\mathrm{GHz})$ & \% of lost packets & Average RRT (ms) \\
\hline 1 Mbps & $\mathbf{2 . 4 2 2}$ and $\mathbf{2 . 4 3 2}$ & $20 \%$ to $30 \%$ & 28 and 20 \\
$2 \mathrm{Mbps}$ & $\mathbf{2 . 4 1 7}, \mathbf{2 . 4 2 2}$ and $\mathbf{2 . 4 3 2}$ & $10 \%$ to $20 \%$ & 18,20 and 7 \\
$5.5 \mathrm{Mbps}$ & $\mathbf{2 . 4 1 2}$ and $\mathbf{2 . 4 1 7}$ & $40 \%$ to $50 \%$ & 204 and 25 \\
$11 \mathrm{Mbps}$ & $\mathbf{2 . 4 1 7}$ and $\mathbf{2 . 4 2 2}$ & $10 \%$ to $20 \%$ & 24,208 and 547 \\
\hline
\end{tabular}

Table 3. Summary of results for $17 \mathrm{~cm}$.

\begin{tabular}{llll}
\hline Modulation & Best frequencies $(\mathrm{GHz})$ & \% of lost packets & Average RRT (ms) \\
\hline 1 Mbps & $\mathbf{2 . 4 2 7}$ & $40 \%$ & 28 \\
$2 \mathrm{Mbps}$ & $\mathbf{2 . 4 2 2}$ & $30 \%$ & 46 \\
$5.5 \mathrm{Mbps}$ & $\mathbf{2 . 4 2 7}$ & $50 \%$ & 3 \\
$11 \mathrm{Mbps}$ & $\mathbf{2 . 4 2 7}$ & $70 \%$ & 17 \\
\hline
\end{tabular}

As we can see, the amount of lost packets and average value of RTT does not affect them equally at all frequencies. The performance worsens starting from $2.432 \mathrm{GHz}$ to upper frequency values. However, the first frequency does not present a notable degradation of the performance with the increase of frequency.

\section{Conclusion}

Research on underwater communications and the use of Underwater Wireless Sensor Networks are becoming a very hot topic because of the appearance of new marine/oceanographic applications. Communications based on EM wave transmission offer great benefits such as the increase of the bandwidth of the link to transmit more information.

In this paper, we performed several tests at different frequencies and modulations to check several parameters such as the minimum depth, distance between devices and signal transmission characteristics. These tests have been done in the first seven frequencies that are specified in the IEEE 802.11 standard.

We note several factors. On the one hand, we see that the modulation (thus the data transfer rates) that show better performance are BPSK and QPSK, with percentage of lost packets lower than $30 \%$ for distances up to $16 \mathrm{~cm}$. For $17 \mathrm{~cm}$, we also obtained a percentage of lost packets of $30 \%$ when QPSK modulation is used. In addition, we observed that the RTT values for $16 \mathrm{~cm}$ are around $25 \mathrm{~ms}$, when the system was working at $2.432 \mathrm{GHz}$. Thus, contrary to what we initially thought (the higher frequency, the higher attenuation), it seems that the global system performance improves slightly when it works at $2.432 \mathrm{GHz}$, compared with the results of the measurements obtained when it is working at $2.412 \mathrm{GHz}$.

As we have told, due to our proposal provides short communication distances in UWSN, it is easy to think that because the water has a high attenuation of these frequencies, underwater communications in the $2.4 \mathrm{GHz}$ band, is unhelpful and impractical. 
However, there are very specific applications where the use of EM waves to transmit information at very short distances, offer great benefits. We can use it, for precision monitoring such as ecosystems contaminated by invasive plants (especially in ponds where there are some poisonous plants that can contaminate the water) or hazardous waste (e.g. in swamps, the quality of the water is different depending on the season because the water may contain some organic material that may be affected when it is warmer because the $\mathrm{pH}$ is different). In both cases the water cannot be used for human consumption, but, in some cases, it can be used by industries to run their plants and supply the water cooling system.

We also would like to use this underwater communication system in the neutrino telescope project [18]. The neutrino telescope is an underwater structure located at the bottom of the Mediterranean Sea. This system allows the detection of cosmic particles, as neutrinos. It consists of thousands of optical detectors and photomultipliers, which must communicate with other system parts, located at distances, extremely small (practically in contact). The photodetectors are distributed in threes along umbilical cables of 450 meters high, designed to carry signals and power. Until now they are using cables and penetrators, to unite the different parts. These pieces have a high economic cost. Using wireless communications, we would be reducing the cost of this material and would avoid the critical connections that can propagate a fault (or leak) through the system. Finally, the fact that the distances between the devices are so small, makes the depth of this infrastructure is not a problem for the transmission of information. There are other applications such as, military applications, marine monitoring and even industrial applications such as marine fish farms [19], to reduce the deposition of organic waste on the seabed and to fight against environmental contamination.

We want to extend the applicability of this system. To do this, our next studies will be focused in two directions. The first will focus on gradually reducing the work frequency, trying to keep the values of transmission. The second line of research will be the design of an antenna optimized for underwater transmission of EM signals in the frequency band of $2.4 \mathrm{GHz}$ and other inferior frequencies that we can prove in the study.

\section{Acknowledgements}

This work has been partially supported by the "Ministerio de Ciencia e Innovación", through the "Plan Nacional de I+D+i 2008-2011" in the "Subprograma de Proyectos de Investigación Fundamental", project TEC2011-27516, and by the Polytechnic University of Valencia, though the PAID-15-11 multidisciplinary projects.

\section{References}

1. Mohsin, A.H., Bakar, K. A., Adekiigbe, A., Ghafoor, K.Z., A Survey of Energy-aware Routing protocols in Mobile Ad-hoc Networks: Trends and Challenges, Network Protocols and Algorithms. 2011 Vol. 3, No. 4, Pp. 1-17 
2. Segal, M. Improving Lifetime of Wireless Sensor Networks, Network Protocols and Algorithms. 2009, Vol. 1, No. 2, Pp.48-60

3. Garcia, M., Sendra, S., Atenas, M., and Lloret, J., Underwater Wireless Ad-hoc Networks: a Survey. Book: Mobile Ad hoc Networks: Current Status and Future Trends, CRC Press. 2011, Pp. 379-411.

4. Chakraborty, U., Tewary, T., and Chatterjee, R.P., Exploiting the loss-frequency relationship using RF communication in Underwater communication networks. In proceedings of 4th International Conference on Computers and Devices for Communication, CODEC 2009, Kolkata, India, December 14-16, 2009.

5. Liebe, H.J., Hufford, G.A., and Manabe; T., A model for the complex permittivity of water at frequencies below $1 \mathrm{THz}$. International Journal of Infrared and Millimeter Waves. 1991, Vol.12, No.7, Pp. 659-675.

6. Somaraju, R., and Trumpf, J., Frequency, Temperature and Salinity Variation of the Permittivity of Seawater. IEEE Transactions Antennas and Propagation. 2006, Vol.54, No. 11, Pp. 3441-3448.

7. IEEE Std 802.11 (2007) IEEE Standard for Information technology-telecommunications and information exchange between systems - Local and metropolitan area networks-Specific requirements-Part 11: Wireless LAN Medium Access Control (MAC) and Physical Laye (PHY) Specifications. Pp. 1-1184. New York, USA

8. Chaitanya, D.E, Sridevi, C.V, and Rao, G.S.B., Path loss analysis of underwater communication systems, 2011 IEEE Students' Technology Symposium (TechSym 2011), Kharagpur (India), pp.65-70, January 14-16, 2011.

9. Sehgal, A., Tumar, I., and Schonwalder, J., Variability of available capacity due to the effects of depth and temperature in the underwater acoustic communication channel, OCEANS 2009 - EUROPE. Bremen (Germany), pp.1-6, May 11-14, 2009.

10. Arnon, S., Underwater optical wireless communication network, Journal of Optical Engineering, Vol. 49, 015001. Jan 15, 2010; doi:10.1117/1.3280288

11. Hunt, K.P.; Niemeier, J.J., and Kruger, A., RF communications in underwater wireless sensor networks, IEEE International Conference on Electro/Information Technology 2010, Normal, Illinois (USA), May 20-22, 2010.

12. Jiang, S., and Georgakopoulos, S., Electromagnetic Wave Propagation into Fresh Water, Journal of Electromagnetic Analysis and Applications, Vol.3, No.07, Pp. 261-266, Jun. 2011.

13. Sendra, S., Lamparero, J.V., Lloret, J., Ardid, M., Underwater Communications in Wireless Sensor Networks using WLAN at 2,4Ghz. International Workshop on Marine Sensors and Systems (MARSS), Valencia (Spain), October 17-22, 2011.

14. Martin, F., Gorday, P., Adams, J., and Leeuwen, H. V., IEEE 802.15.4 PHY Capabilities. Doc.: 15-04-0227-04-004A. May 2004. Available at: https://mentor.ieee.org/802.15/file/04/1504-0227-04-004a-ieee-802-15-4-phy-layer-and-implementation.ppt

15. Chitode, J. S., Digital Communications, Technical Publications Pune. First edition: 20072008.

16. Andren, C., and Webster, M., CCK Modulation Delivers 11Mbps for High Rate 802.11 Extension. In Proceedings of the Wireless Symposium/Portable By Design Conference, Spring, San Jose, CA, USA, February 22 - 261999.

17. Lloret, J., López, J. J., and Ramos, G., Wireless LAN Deployment in Large Extension Areas: The Case of a University Campus, In proceedings of Communication Systems and Networks 2003, Benalmádena, Málaga (Spain), September 8-10, 2003.

18. Ardid, M., ANTARES: An Underwater Network of Sensors for Neutrino Astronomy and Deep-Sea Research, Ad Hoc \& Sensor Wireless Networks, 2009. Vol. 8, Pp. 21-34.

19. Garcia, M.; Sendra, S.; Lloret, G and Lloret, J. Monitoring and Control Sensor System for Fish Feeding in Marine Fish Farms, IET Communications, The Institution of Engineering and Technology. August 2011. Vol. 5, No. 12, Pp.1682 - 1690. 